\title{
A comunicação matemática por meio das tarefas de investigação: caminhos alternativos para o ensino e aprendizagem de matemática
}

\author{
Mathematical communication through research tasks: \\ alternative ways for teaching mathematics
}

Comunicación matemática a través de tareas de investigación: caminos alternativos para la enseñanza y el aprendizaje de las matemáticas

\author{
Benedito Edson Cardoso Machado \\ Universidade Federal do Pará - UFPA \\ Breves, Pará, Brasil \\ E-mail: edson.cardoso.oficial.pa@gmail.com \\ Orcid:0000-0001-5362-5707 \\ Alan Gonçalves Lacerda \\ Universidade Federal do Pará- UFPA \\ Breves, Pará, Brasil \\ E-mail: alanlacerda@ufpa.br \\ Orcid:0000-0002-7447-7683
}

\begin{abstract}
Resumo: Neste trabalho objetivamos analisar e discutir as ações comunicativas e as tarefas de investigação para o ensino de matemática. Trouxemos como aporte teórico Rodrigues, Menezes e Ponte (2018); Bondie \& Zusho (2016); Guerreiro (2014); Menezes et al (2014); Alrø \& Skovsmose (2010). Para a metodologia, apresentamos e discutimos três tarefas investigativas: "Cubos com autocolantes", "Dobras e mais dobras" e "Sequencia pictórica". Os resultados apontam que as tarefas de investigações explorada por meio da comunicação matemática contribuem para o processo de ensino e de aprendizagem trazendo uma criticidade sobre as tarefas, além de contribuir para as exposições de ideias e defesas dos pontos de vista dos educandos.
\end{abstract}




\begin{abstract}
In this work we aim to analyze and discuss communicative actions and research tasks for teaching mathematics. We brought as theoretical contribution Rodrigues, Menezes and Ponte (2018); Bondie and Zusho (2016); Warrior (2014); Menezes et al (2014); Alrø and Skovsmose (2010). For the methodology, we present and discuss three investigative tasks: "Cubes with stickers", "Folds and more folds" and "Pictorial sequence". The results show that the investigative tasks explored through mathematical communication contribute to the teaching and learning process, bringing a criticality to the tasks, in addition to contributing to the exposition of ideas and defenses from the students' points of view.
\end{abstract}

Keywords: Communiation. Tasks, Teaching. Learning.

Resumen: En este trabajo pretendemos analizar y discutir acciones comunicativas y tareas de investigación para la enseñanza de las matemáticas. Trajimos como aporte teórico a Rodrigues, Menezes y Ponte (2018); Bondie y Zusho (2016); Guerrero (2014); Menezes et al (2014); Alro y Skovsmose (2010). Para la metodología, presentamos y comentamos tres tareas investigativas: "Cubos con pegatinas", "Pliegues y más pliegues" y "Pictograma secuencial". Los resultados muestran que las tareas investigativas exploradas a través de la comunicación matemática contribuyen al proceso de enseñanza y aprendizaje, aportando una criticidad sobre las tareas, además de contribuir a la exposición de ideas y defensas desde el punto de vista de los estudiantes.

Palabras clave: Comunicación. Tareas. Enseñando. Aprendiendo.

Recebido em 03/06/2020

Aceito em 24/03/2021 


\section{INTRODUÇÃO}

A comunicação matemática tem motivado 0 interesse de diversos pesquisadores no campo da Educação Matemática (Machado \& Lacerda, 2020; Rodrigues, Menezes \& Ponte, 2018; Bondie \& Zusho, 2016; Menezes \& Delplancq, 2016; Ponte, 2014; Menezes et al, 2014; Alrø \& Skovsmose, 2010; Ponte, 2003; 2002; Ponte \& Serrazina, 2000). Esses autores são unanimes na coroação de experiências de práticas bem sucedidas para a sala de aula promulgada sob o enfoque da comunicação. Das ações comunicativas oriundas que recomendam estes autores seguem algumas: exploração de tarefas matemáticas, formulação de conjecturas, defesas dos pontos de vistas e interação social.

No que diz respeito às tarefas Ponte (2014) apresenta quatro: 1) exercício; 2) problema; 3) exploração e 4) investigação. Para tanto, para esse estudo nos delimitaremos e nos reportaremos às tarefas investigativas. $\mathrm{Da}$ qual problematizaremos: Quais tarefas investigativas podem apresentar caminhos para a exploração de ações comunicativas na sala de aula de matemática?

O presente questionamento integrou uma ação de preparação sobre como conectar e desenvolver atividades para subsidiar as aulas do professor de matemática. Para alcançar tais estudos foram explanadas atividades juntos aos participantes do minicurso realizado por Machado e Lacerda (2019), tendo como sujeitos participantes professores e licenciandos em matemática, mas também relacionados a exemplos com o ensino.

Dessa forma, vislumbraremos estudos na literatura especializada que evidenciam as tarefas de investigação com o objetivo de integrar um corpo relativamente delicado e essencial que é a formulação da pergunta.

\section{O EMERGIR DA COMUNICAÇÃO MATEMÁTICA MEDIANTE AS TAREFAS INVESTIGATIVAS}

Comunicar na sala de aula é possibilitar a troca de informações entre os interlocutores (professor e aluno) em busca de um entendimento, um sentido e 


\section{Universidade Federal da Grande Dourados}

significado para tarefa. Menezes et al (2014, p. 136) descreve que "a comunicação é um elemento essencial nas práticas letivas dos professores", esta comunicação estar presente na vida de todos os professores. Entretanto, buscamos analisar algumas ações que podem contribuir ainda mais para o processo de ensino e o processo de aprendizagem.

Quando falamos em comunicação matemática, pensamos no sentido que versa Ponte (2014) que nos sugere como partilhas de informações, exposições de ideias, conjecturas e defesas de pontos de vistas. Além de auxiliar os professores no desenvolvimento e planejamento de tarefas e, consequentemente, no insight, mas também garantir o crescimento dos alunos envolvidos na tarefa.

Em outra perspectiva, entendemos que existam uma diversidade de modelos que possam ser implementados no cotidiano escolar, bem como diferentes daqueles tradicionalmente evidenciados nas aulas, tal como o modelo sanduíche descritos por Alrø \& Skovsmose (2010) quando o professor faz uma pergunta, o aluno responde e o professor avalia.

Para Menezes (1996, p. 22), o modelo comunicativo que deve ser promulgado "é aquela em que alunos e professor alternam os papeis de emissor e receptor", bem como, partilham novas ideias e defesas de seus pontos de vistas. Nesse sentido, Rodrigues, Menezes \& Ponte (2014) descrevem que esse modelo de partilha de ideias, novos significados e questionamentos, colocam grandes desafios para esses interlocutores, mas também, grandes contribuições para o processo de ensino e de aprendizagem.

Diante disso, ações comunicativas podem ser evidenciadas pelos professores de matemática para que de fato a comunicação matemática passe a ser bem potencialidade na sala de aula. Ponte \& Serrazina (2000) destacam três ações comunicativas fundamentais na sala de aula de matemática que podem ser exploradas: a exposição de ideias (primeira ação) possibilitaria aos alunos e ao professor a expor suas formas de pensar. Seguido pelos questionamentos (segunda ação) que poderiam surgir ou os professores e alunos poderiam criá-los, e no final, ter uma discussão (terceira ação) sobre as conclusões e apresentações dos resultados 


\section{Universidade Federal da Grande Dourados}

encontrados e defendê-los perante os demais colegas, e estes possam ouvir e/ou contribuir para a tarefa em questão.

Ainda se referindo a esses autores, as exposições podem ser apresentadas mediantes um relato de uma história, de uma experiência, comumente realizada em sala de aula. Entendemos que essa exposição é importante para que possamos ter uma aula de modelo comunicativo, pois acaba "quebrando o gelo" e os alunos passam exporem suas opiniões e questionamentos.

Em relação aos questionamentos (segundo ato comunicativo), também é uma prática muito comum na vida de qualquer professor, pois é o momento em que realizam perguntas aos seus alunos. Entretanto, para a potencialidade do modelo comunicativo, não é qualquer pergunta que possam vim contribuir para o processo de ensino e de aprendizagem nesse modelo. Dessa forma, autores como Pereira (1991), Cohen \& Manion (1992), Love \& Mason (1995) e Bondie \& Zusho (2016) descrevem algumas características de perguntas que podem contribuir para a sala de aula e sugerimos a essa para a otimização dos processos de gerar insights.

Para Pereira (1991) citado por Menezes (1996) quatro são as finalidades das perguntas: (i) centrar a atenção dos alunos em aspectos que o professor considera relevante; (ii) provocar efeitos positivos na participação dos alunos (fazê-lo falar); (iii) promover no aluno uma atitude intelectual menos passiva (fazê-lo pensar); e (iv) minimizar o efeito da indisciplina. Para o modelo comunicativo, acreditamos que as três finalidades inicias podem promover essa comunicação na sala de aula. Já Cohen e Manion (1992) descrevem outras duas: (i) fazer pensar os alunos e (ii) testar o conhecimento constantemente.

Nesse escopo, Bondie \& Zusho (2016) acreditam que as perguntas devem atingir alguns objetivos para que possam contribuir para o processo de ensino e de aprendizagem, dentre esses objetivos, os autores apresentam três: (i) obter informação; (ii) construir compreensão e (iii) encorajar reflexões.

Para Mata (1990, apud Menezes, 1996) o professor realiza as perguntas focadas em outra vertente: (i) Obter informação que não possui; (ii) provocar indiretamente a realização de ações; (iii) orientar os alunos na sistematização da 


\section{Universidade Federal da Grande Dourados}

informação relativa e; (iv) avaliar a quantidade e a qualidade do conhecimento dos alunos.

Já Love \& Mason (1995) descrevem as perguntas em três frentes e as denomina como: perguntas de inquirição, estas são realizadas com objetivo de obter informação dos alunos em seus processos; as perguntas de focalização, estas podem focar as atenções dos alunos em contextos específicos; já as perguntas de confirmação, buscam confirmar algo que o aluno já sabe ou simplesmente, testa o conhecimento do educando.

Nos estudos de Machado \& Lacerda (2020) em que buscaram identificar às perguntas que poderiam contribuir ao processo de ensino e aprendizagem mediante o desenvolvimento de uma tarefa exploratório-investigativa pontou que há aspectos essenciais nas perguntas que potencializam as ações entre os interlocutores (professor-aluno e aluno-aluno).

Deste modo, quando as perguntas são realizadas buscando esses objetivos, podemos analisar as suas potencialidades encorajando os alunos a novas descobertas, construindo novas conjecturas. Nessa ação comunicativa, as perguntas que são realizadas pelos professores com o intuito de explorar as potencialidades, não terão uma resposta de antemão, eles passarão em conjuntos com os alunos e encontrar novas estratégias de resolução para os novos questionamentos.

Mediante as exposições de ideias (primeiro ato comunicativo) e questionamentos (segundo ato comunicativo) presente no modelo comunicativo, a discussões (terceiro ato comunicativo) ganha um espaço de destaque dentro da sala de aula de matemática. Segundo Ponte \& Serrazina (2000) a discussão pode assumir as interações entre os interlocutores, estes podem se alternar expondo suas ideias e/ou questionando.

Rodrigues, Menezes \& Ponte (2018, p. 399) entendem que "as discussões são momentos de trabalho na sala de aula com grandes potencialidades para a aprendizagem dos alunos, ao favorecerem o seu desenvolvimento na apresentação, justificação, argumentação e negociação de significados", nesse processo de 


\section{Universidade Federal da Grande Dourados}

discussão os alunos podem defender as suas estratégias encontrando novos caminhos para a resolução de problemas.

Afirma Rodrigues, Menezes \& Ponte (2014, p. 66) que "na condução de uma discussão coletiva, o professor é chamado a desempenhar diversas funções que são influenciadas e influenciam o seu conhecimento" e estas funções buscam contribuir para o processo de aprendizagem.

Ponte (2005) acrescenta ainda que uma discussão tem sempre um objetivo, por exemplo, a estratégia para a realização de uma tarefa, a avaliação de uma dada solução e o balanço do trabalho realizado ao longo de todo um período, estas avaliações e estratégias expostas são analisadas pelos demais alunos e professor que podem ainda questionar e solicitar ao interlocutor a defender a sua ideia.

As discussões de resultados apresentam-se normalmente após o termino de uma tarefa em que os alunos as desenvolvem. Para estes estudos, nos desdobraremos sobre as tarefas de investigação que são campos de estudos de Ponte (2014) e Alrø \& Skovsmose (2010). Entendemos que este tipo de tarefa é eminente para a exploração das ações comunicativas na sala de aula de matemática.

Ponte, Brocardo \& Oliveira (2013, p. 23) enfatizam que:

O conceito de investigação matemática, como atividade de ensinoaprendizagem, ajuda a trazer para a sala de aula o espírito da atividade Matemática genuína, constituindo, por isso, uma poderosa metáfora educativa. O aluno é chamado a agir como um matemático, não só na formulação de questões e conjecturas e na realização de provas e refutações, mas também na apresentação de resultados e na discussão e argumentação com os seus colegas e o professor.

O conceito de investigação na matemática ainda é muito recente no âmbito da educação Matemática. Investigar é possibilitar os alunos a novas descobertas, criar novas estratégias, criar conjecturas. Investigar possibilita os alunos a serem matemáticos em busca do desconhecido, em busca novos caminhos de resoluções (PONTE, 2014). 


\section{Universidade Federal da Grande Dourados}

Ponte (2014) diz que uma tarefa investigativa é aquela que tem um grau de desafio elevado, ou seja, aquela que o aluno tem mais dificuldade em sua resolução. Também acrescenta que uma tarefa investigativa tem um grau de estrutura mais aberto, ou seja, podemos identificar essa estrutura conforme a elaboração do enunciado, para este autor, os enunciados desse tipo de tarefa não contêm muitas informações pois o objetivo é que os alunos possam a investigar e encontrar novas resoluções para esta tarefa, já que esta não tem uma única resposta.

Já para Fonseca, Brunheira \& Ponte (1999, p. 4), a tarefa investigativa é realizada com o objetivo de "explorar todos os caminhos que surgem como interessantes a partir de uma dada situação. É um processo divergente. Sabe-se qual é o ponto de partida, mas não se sabe qual será o ponto de chegada". Dessa forma, compreendemos que uma tarefa investigativa proporciona aos alunos a serem construtores dos seus conhecimentos e chegar a um resultado.

Em outra percepção, os autores Araújo \& Ferreira (2019, p. 6) com enfoque no ensino, consideram que:

O conhecimento não é algo pronto e acabado, pois por meio da procura por soluções de uma atividade o aluno pode chegar ao resultado esperado, porém por caminhos diferentes tanto em relação ao pensado pelo professor como pelo apontados por seus colegas de classes.

Nessa percepção, as tarefas investigativas tem um ponto de partida na qual o professor imagina qual seria o resultado, porém como a tarefa investigativa é caracterizada com estrutura aberta, logo os alunos podem percorrer caminhos diferentes, e estes caminhos podem contribuir para o processo de ensino.

Dentro dessa vertente, os professores que adotarem esse modelo de ensino sairiam de suas zonas de conforto, pois não saberiam as respostas dos alunos de antemão, dessa forma entrariam numa situação em que Alrø \& Skovsmose (2010) descrevem como a zona de risco, pois estes educadores não teriam esse controle habitual da turma e passariam juntos com os alunos encontrar novas estratégias de soluções. Diante disso, entendemos os desafios que os professores enfrentariam 


\section{Universidade Federal da Grande Dourados}

adentrando nesse modelo, porém buscamos ressaltar que as potencialidades que surgem nesse modelo contribuem para a formação do educando.

Para os autores Pereira \& Saraiva (2005, p. 44) as tarefas de investigação:

Permite a exploração de situações através de construções visuais e analíticas, importantes e uteis ao próprio processo de criação matemática. Por outro lado, permite a extensão da linguagem natural à linguagem matemática, através de representações simbólicas de conceitos abstractos e da sua consequente articulação e comunicação.

Durante as tarefas de investigação a comunicação matemática emerge por meio da linguagem natural e a linguagem matemática, proporcionando aos alunos a encontrar novos sentidos e significados. Deste como, a comunicação pode ser explorada solicitando aos alunos a exporem suas ideias, questionamentos e no final realizar uma discussão sobre os resultados que cada aluno chegou, ou seja, realizar uma socialização sobre a tarefa investigativa realizada.

Dessa forma, a comunicação é explorada com o objetivo de contribuir para o processo de ensino e aprendizagem. Os professores podem expor suas opiniões, formular perguntas com intuito de obter informações (MACHADO; LACERDA, 2019; PEREIRA, 1991; COHEN; MANION, 1992; LOVE; MASON, 1995; BONDIE; ZUSHO, 2016) e proporcionar a discussão na sala de aula.

Para Martins \& Guerreiro (2017) a comunicação como interação entre os alunos e como instrumento do professor podem ser pressupostos para a construção do conhecimento, através do ensino e da partilha de conhecimentos matemáticos, bem como as tarefas de investigações como ferramenta de exploração da comunicação na sala de aula de matemática.

\section{EXPLICAÇÕES E EXEMPLOS}

Dentre essas perspectivas de análises e discussões, vamos assinalar caminhos que podem contribuir para a prática docente, bem como para a exploração 
da comunicação. Sendo assim, a seguir apresentaremos alguns exemplos de atividades e seus desdobramentos.

Tarefa "Cubo com autocolantes"

A presente tarefa que foi desenvolvida por Ponte e seus colaboradores (2014), tem como intuito analisar a quantidade de autocolantes (adesivos) podes ser colocado em um cubo que estavam posicionados da seguinte forma. (figura 1)

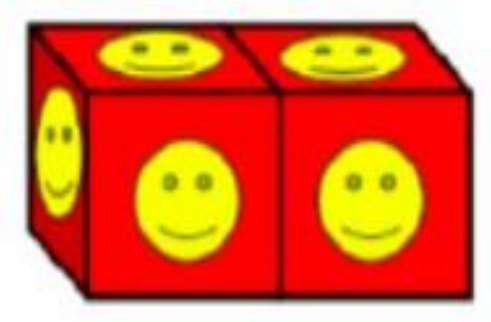

Figura 1 - cubo com autocolantes

Fonte: Ponte et al (2014)

Como já sabemos, um cubo tem seis fáceis, logo, seriam necessários seis autocolantes para todas as faces desse cubo. Suponhamos que uma dessas faces esteja colada no cubo seguinte, desse modo, não seria possível cola esses autocolantes em algumas dessas fáceis.

Para o contexto da sala de aula, o professor-pesquisador pode realizar uma exposição de ideias por meio de uma pergunta, por exemplo: "Não seria legal se soubéssemos quantos autocolantes precisaríamos para cola em um cubo?". Esse tipo de situação convidativa segundo Alrø \& Skovsmose (2010) trazem a investigação um convite para que os alunos possam se sentir à vontade, e não serem obrigados a participarem. Percebemos que, ao questionar, o professor incentiva os alunos a buscarem resoluções.

Love \& Mason (1995) enfatizam as perguntas buscam obter informações dos alunos, podendo contribuir para o processo de ensino e aprendizagem. Para ser mais incisivo, o professor-pesquisador pode realizar a seguinte pergunta " $E$ se quiséssemos saber quantos autocolantes precisaria para cola em 20 cubos?", essas perguntas colocaria os alunos no papel de investigador e este, podendo encontrar formas de resolução. 
Para encerrar a tarefa, o professor-pesquisador pode solicitar aos alunos a realizar uma socialização de seus resultados, para que os demais alunos da turma e o professor possam saber quais estratégias criadas. Para Rodrigues, Menezes \& Ponte (2018) a discussão na sala de aula de matemática contribui para a compreensão, pois os alunos passam a defender suas ideias matematicamente.

Diante disso, podemos perceber que são inúmeras estratégias que os alunos podem desenvolver no decorrer da tarefa, possibilitando aos alunos a apresentarem suas formas de resolução perante a turma e defender sua forma de resolução. A seguir, vamos expor a segunda tarefa denominada "dobras e mais dobras".

\section{Tarefa "Dobras e mais dobras"}

A tarefa "dobras e mais dobras" realizado por Ponte e seus colabores (2014), proporciona os alunos a realizarem pequenas dobras de um papel A4 e representarem matematicamente a quantidade dessas dobras. (Conforme a figura 2)

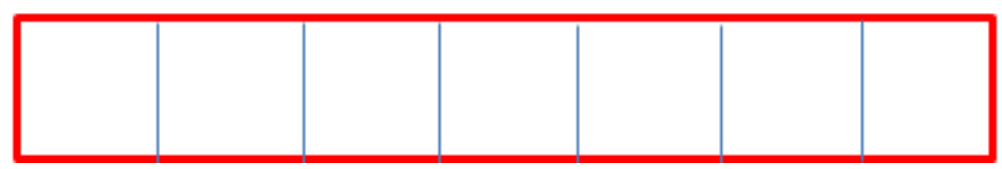

Figura 2 - Dobra de papel

Fonte: Adaptado de Ponte et al (2014)

Nesta tarefa, podemos analisar uma representação de um pedaço de papel que foi dobrado em sete partes (suponhamos que essas listras azuis sejam dobras desse papel retangular de bordas vermelhas). Dessa forma, o professor-pesquisador pode busca diferentes desdobramentos de como proceder essa tarefa, especialmente para essa tarefa, o professor pode incentivar os alunos por meio de perguntas.

O professor-pesquisador pode realizar perguntas aos alunos fazendo com que eles representam essas dobragens da forma que quiserem, como por exemplo. Os alunos podem representar estratégias diversas formas por meio de porcentagens ou em formas de frações, bem como ainda em linguagem matemática ou mesmo em linguagem coloquial como "um pedaço de sete". 


\section{Universidade Federal da Grande Dourados}

Nesse sentido, poderíamos perceber as inúmeras formas de representar e explorar as dobras de um pedaço de papel dentro do contexto da sala de aula. Para Kieren (1988, apud SILVA, 2015, p. 29) "o modelo parte-todo para o ensino dos números fracionários auxilia convenientemente na produção da linguagem fracionaria, quando os textos de matemática da escola e o discurso do professor, tendem a orientar o estudante a uma imagem de dupla contagem".

Diante desse exposto, a linguagem materna pode contribuir para o ensino e aprendizagem nas representações desses números, especialmente, as representações dessa dobragem de papel.

Nessa perspectiva, os alunos podem desenvolver a linguagem natural e a linguagem matemática. Para Menezes (2014) essas linguagens destacam a progressiva clarificação entre esses dois modelos comunicativos na qual emergem a negociação de significados. Desta forma, quando ocorre essa negociação pode contribui para o entendimento dos conteúdos matemática.

Diante disso, o professor-pesquisador pode realizar com os alunos etapas, por exemplo, solicitar aos alunos a dobrou um papel ao meio e representa-la matematicamente. E, seguida, dobrar novamente e escrever sua representação. Desta forma, os alunos poderiam dobra esse papel em diversas vezes e representalo.

Desta forma, alguns desdobramentos mediante a tarefa podem ser elencados, como na introdução da tarefa o professor pode emergir uma pergunta. Para Ponte (2014) a introdução de um assunto é importante para que os alunos possam ter familiaridade com a tarefa, para que estes possam se sentir mais à vontade.

Para o desenvolvimento, o professor-pesquisador pode realizar pergunta, exemplos, "Como podemos representar uma laranja repartida ao meio?". No questionamento o professor pode solicitar opiniões dos alunos. Love \& Mason (1995) apresentam alguns tipos de perguntas, como as perguntas de Inquirição, confirmação e focalização. As perguntas de confirmações são realizadas com o objetivo de confirmação um resultado, muitas das vezes é uma pergunta retorica, simplesmente para testa o conhecimento do aluno ou para chamar a sua atenção. A pergunta de 


\section{Universidade Federal da Grande Dourados}

focalização é realizada com o intuito de focar a atenção do aluno em um determinado contexto especifico na qual o professor gostaria de abordar para o enriquecimento do determinado problema.

Para a exploração da comunicação, enfatizamos as perguntas de inquirição que potencializam ainda mais a tarefa, pois estas perguntas buscam cada vez mais obter informação dos alunos.

Para a finalização dessa tarefa, o professor pode chamar os alunos a mostrarem aos seus colegas as suas formas de apresentações. Esse momento de discussão pode possibilita os alunos uma compreensão das diversas formas de representações, desenvolvendo assim, a linguagem matemática. A seguir, apresentaremos a última tarefa, sequências pictóricas.

Tarefa "Sequências pictóricas"

A tarefa investigativa denominada "sequência pictórica", foi realizada pelos autores Ponte e seus colaboradores (2014) utilizando palitos de fósforos para construírem um triangulo (como mostra a figura 2), tendo como objetivo encontrar a quantidade de números de palitos em um determinado número de triângulos.
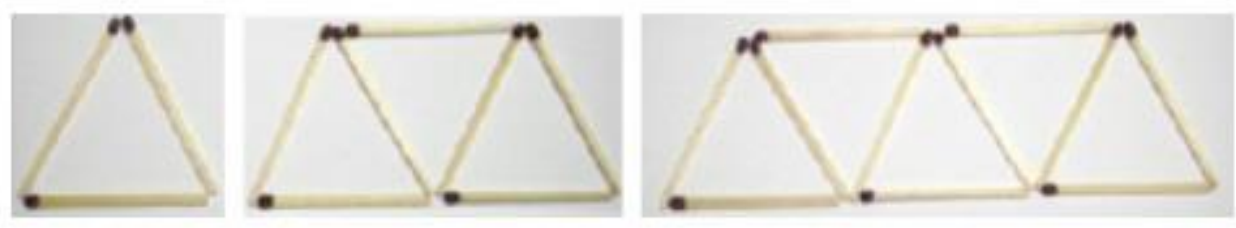

Figura 2 - Sequência pictórica

Fonte: Ponte et al (2014)

Nesse sentido, os alunos podem construir novos triângulos, dando continuidade à sequencias. Nesse viés, o professor-pesquisador pode instigar os alunos a encontrarem os números de palitos necessários para construir um determinado número de triangulo ou para um determinado número de triangulo são necessários quantos palitos. O professor-pesquisador pode ainda solicitar aos alunos a construírem uma formula para determinar essa sequência. 


\section{Universidade Federal da Grande Dourados}

Durante a tarefa, o professor pode realizar questionamento, como, "Será que podemos descobrir quantos palitos são necessários ao chegar no 10 triangulo nessa sequência?" e "e se a gente pudesse construir uma formular para não precisar conferir?". Durante as estratégias formuladas pelos alunos, o professor também pode realizar perguntas do tipo "por que você fez isso?", "como você fez isso?", "pensou nisso como?" e entre outras perguntas.

O NCTM (1994) relata que as perguntas do tipo "Por quê" e o pronome "E se" possibilita os alunos a explicarem e construírem conjecturas, bem como formula estratégias de solução. Para Pereira (1991), uma vez que estas perguntas são realizadas com intuito de provocar efeitos positivos nos alunos, estes efeitos podem ocorrer em suas falas por meio de exposição de ideias, explicações e entre outros.

Para esta tarefa, Ponte e seus colaboradores (2014), apontam duas formas de os alunos conseguirem desenvolver. A primeira formula é $4 \mathrm{n}-1$ e a segunda, é $2 n+(2 n+1)$. Entretanto, como é uma tarefa investigativas os alunos podem formular novas formulas e métodos de resoluções para chegar ao resultado, mas para isso devem ser desafiados pelo professor.

Diante disso podemos perceber que os alunos passam a ser matemáticos em busca de soluções, investigam e defendem suas estratégias e estes passam a ter criticidade sobre o ensino e aprendizagem. Dessa forma, o ensino não é pronto e acabado, os alunos passam a ser construtores do conhecimento matemática, contribuindo assim para o processo de ensino e aprendizagem da matemática. (ARAÚJO \& FERREIRA, 2019)

Diante disso, os alunos podem criar conjecturas, formular equações, criar estratégias, desenvolver a linguagem matemática em busca de novos significados e entre outros. Como podemos analisar, são várias as potencialidades que podem surgir mediante a uma tarefa investigativa que contribui para o processo de ensino e de aprendizagem de matemática.

Universidade Federal da Grande Dourados 


\section{CONSIDERAÇÕES FINAIS}

A comunicação matemática é um campo de estudo crescente na área da Educação Matemática, pois pode ser explorada em diversas situações da sala de aula, claro que para essa comunicação ser bem explorada devem ser exploradas ambientes de investigação que traga contribuições para o processo de ensino e de aprendizagem.

A investigação matemática dentro dessa vertente, apresentasse um caminho que traz contributos para a comunicação matemática, pois a investigação possibilita aos alunos a investigarem e construírem conjecturas para encontrar novas soluções, por conta disso, os alunos passam a se comunicar dentro da sala de aula com os outros colegas e com o professor.

Nesse sentido, as tarefas apresentadas "cubo com autocolantes", "dobras e mais dobras" e "sequência pictórica" foi analisada e discutida com intuito de mostrar que as investigações podem ser um dos caminhos alternativos para a sala de aula na qual podemos contribuir ainda mais ao explorar as ações comunicativas.

Desta forma, durante esse ambiente de aprendizagem o professor pode explorar a comunicação que contribua para o processo de ensino, proporcionando aos alunos a oportunidade de exporem suas ideias, questionamentos e discussões de suas estratégias. Já Para o processo de aprendizagem, os alunos passam a questionar suas resoluções e propor questionamentos, além de construir criticidades por meio da tarefa. Nessas investigações, os alunos passam a ser um matemático, encontrando soluções matematicamente e defendendo as suas ideias frente a turma.

Diante disso, podemos concluir que estas tarefas apresentadas são um dos caminhos alternativos que os professores podem propor na sala de aula de matemática. Com isso, podemos inferir que qual seja a tarefa investigativa vai proporciona aos alunos a investigar, e mediante a investigação o ambiente da sala de aula à comunicação pode ser explorado que potencialize o processo de ensino e aprendizagem. 
Em síntese, durante a tarefa investigativa, a comunicação na sala de aula vem agregar ainda mais quando explorada de maneira que contribua para a compressão dos educandos. Nesse estudo analisamos e discutimos apenas três tarefas, e estas tarefas puderam ser exploradas mediante as perguntas, exposições de ideias e discussões. Sendo assim, as tarefas investigativas assinalam caminhos alternativos para a contribuição para o processo de ensino e de aprendizagem.

\section{REFERÊNCIAS}

Alrø, H., \& Skovsmose, O. (2010). Diálogo e Aprendizagem em Educação Matemática (2a ed.). Belo Horizonte: Autêntica.

Araújo, F. J. M., \& Ferreira, R. S. (2019). O desenvolvimento de conceitos estatísticos por meio da investigação matemática. Tangram - Revista de Educação Matemática, 1(4), 03-18.

Bondie, R., \& Zusho, A. (2016). (Under contract). Book proposal: Engaging the Extremes: Classrom Routines for precise, Efficient, and effective learning for all. Routledge. Recuperado em: http://educarmelhor.com. br/noticia/como-fazerperguntas- propositivas-para-os-alunos-2, Acesso em 16 de setembro de 2019.

Cohen, L., Manion, L., Morrison, K., \& Wyse, D. (2010). A guide to teaching pratice (5a ed.). Londres e New York: Routledge.

Fonseca, H., Brunheira, L., \& Ponte, J. P. (1999). As actividades de investigação, o professor e a aula de matemática. Actas do Profmat 99, Lisboa: APM.

Guerreiro, A. (2014). Comunicação matemática na sala de aula: Conexões entre questionamento, padrões de interação, negociação de significados e normas sociais e sociomatemáticas (Cap. 5, pp. 237-257). Lisboa: Instituto de Educação da Universidade de Lisboa. 
Love, E., Mason, J., Telling \& Asking. (1995). Subject learning in primary curriculum: Issues in English, science and mathematics (1a ed). Londres: Routledge.

Machado, B. E. C., \& Lacerda, A. G. (2020). A comunicação matemática em uma tarefa exploratória- investigativa: uma proposta mediante a taxa de metabolismo basal. Revista De Ensino De Ciências E Matemática, 11(4), 1-21. https://doi.org/10.26843/rencima.v11i4.2469

Machado, B. E. C., \& Lacerda, A. G. (2019). Avaliação da competência comunicativa e a tarefa exploratória-investigativa. Anais do Congresso amazônida marajoara de matemática, Breves, PA, Brasil, 1.

Martins, C., \& Guerreiro, A. (2017). Relações entre avaliação e comunicação: perceções de dois professores de matemática. Revista de Estudios e Investigación en psicologia y Educación, Bragança, 1(6), 395-399.

Menezes, L., Ferreira, R.T., \& Guerreiro. (2014). A comunicação nas práticas letivas dos professores de matemática. In: J. P. PONTE (Org.). Práticas profissionais dos professores de matemática (1a ed.). Lisboa: Instituto de Educação da Universidade de Lisboa.

Menezes, L., \& Delplancq, V. (2016). Investigação sobre a própria prática: dois estudos sobre a comunicação matemática. In: Mesquita, C., Pires, M. V., \& Lopes, R. P. (Eds.). Proceedings of the 1 st International Conference on Teacher Education, Bragança: IPB (pp. 371-377).

Menezes, L. A. (1996). comunicação na aula de matemática. Millenium. Viseu, 20 (3), 20-28.

NCTM. (1994). Normas profissionais para o ensino da Matemática. Lisboa: APM. 
Pereira, A. (1991). Comunicação e ensino das ciências: Contributo para o estudo da pergunta no discurso da aula de ciências do ensino básico (Tese de mestrado). Universidade de Lisboa, Lisboa, Portugal.

Pereira, M., \& Saraiava, M. J. (2005). A integração de tarefas de investigação no ensino e aprendizagem das sucessões. Revista Quadrante, Lisboa, 1(2), 4369.

Ponte, J. P. (2002). Investigar a nossa própria prática. Reflectir e investigar sobre a prática profissional. Lisboa: APM.

Ponte, J. P. (2003). Investigar, Ensinar e Aprender. Actas do Profmat. Lisboa: APM, CD - ROM, (pp. 25-39).

Ponte, J. P. (2005). Gestão Curricular em Matemática. In: (Org.). O professor e o Desenvolvimento Curricular. Lisboa: APM, (pp. 11-34).

Ponte. J. P. (2014). Tarefas no ensino e na aprendizagem da matemática. In: (Org.). Práticas profissionais dos professores de matemática. (1a ed.). Lisboa: Instituto de Educação da Universidade de Lisboa (pp. 13-30).

Ponte, J. P., Brocardo, J., \& Oliveira, H. (2013). Investigações matemáticas na sala de aula (3a ed.). Belo Horizonte: Autêntica.

Ponte, J. P., \& Serrazina, M. L. (2000). Didáctica da Matemática do $1^{\circ}$ Ciclo (1a ed.). Lisboa: Universidade Aberta.

Rodrigues, C., Menezes, L., \& Ponte, J. P. (2014). Práticas de discussão matemática no ensino da álgebra. Anais do Seminário de Investigação em Educação Matemática. Braga, Portugal, 25. 
Rodrigues, C., Menezes, L., \& Ponte, J. P. (2018). Práticas de discussão em sala de aula de matemática: os casos de dois professores. São Paulo: Bolema, 32 (61), 398- 418.

Silva, M. J. F. (2015). Investigando Saberes de Professores do ensino Fundamental com Enfoque em números fracionários para a quinta série. (Doutorado em Educação Matemática). Pontifícia Universidade católica - PUC-SP, São Paula, SP, Brasil. 